\title{
Lenguas de comunicación o de negocios. La aporta- ción contemporánea del bilingüismo crítico a la enseñanza de lenguas
}

\author{
Carlos E. Segade Alonso \\ Facultad de Ciencias de la Salud y de la Educación \\ Universidad a Distancia de Madrid (UDIMA)
}

Enviado: 30 enero 2014 / Aceptado: 14 diciembre 2014

ISBN: $1697-7467$

\begin{abstract}
RESUMEN: El aprendizaje de la lengua extranjera es asumido por la población como un imperativo, no solo legal, sino social. Esto tiene como consecuencia que la lengua se vea como un bien de alto valor simbólico que hay que lograr poseer para medrar socialmente y no como una parte de la formación para el desarrollo personal. En este artículo cuestiono el modo en cómo se acepta la hegemonía de la lengua extranjera y su valor como capital lingüístico. La teoría crítica aplicada al bilingüismo le otorga un nuevo enfoque personal y social, de forma que la enseñanza de la lengua se enmarca en una didáctica dialógica a partir de principios personalizadores cuyo fin será un proceso equivalente al de una segunda alfabetización.

Palabras clave: lenguas extranjeras, segunda lengua, bilingüismo, teoría crítica, políticas culturales.
\end{abstract}

\section{Communication or Business Languages. Today's Contribution of Critical Bilingualism to Language Teaching}

\begin{abstract}
Foreign language learning is considered by the members of the public as an indisputable imperative, not only legally, but also socially. As a consequence, languages are regarded as assets with a high symbolic value to be obtained in order to socially prosper and not as a part of the process of becoming a person. In this paper I question the way in which the members of the public accept foreign languages' hegemony and its value as linguistic capital. The critical theory applied to bilingualism will provide it with its personal and social approach. Language teaching is part of a dialogical didactics approach that follows personalization principles whose aims are equivalent to those of a second literacy period.

Keywords: foreign languages, second language, bilingualism, critical theory, cultural policies.
\end{abstract}

\section{INTRODUCCIÓN}

No parece que el debate sobre la importancia de algunas lenguas, históricamente hablando, se haya separado nunca del papel más o menos hegemónico que han ido ejerciendo como lenguas de dominio o de poder (Lorenzo, Trujillo y Vez, 2011:48). La Lingüística en el siglo XX comprendió que su objeto de estudio también eran los juegos de poder, las proyecciones en la lengua de situaciones de abuso o de privilegio, junto con la eliminación 
de las diferencias culturales entre lenguas, entrando así en debates sociopolíticos (Lorenzo et al., 2011:57) que más tenían que ver con posturas ideológicas preconcebidas que con el modo en que el uso de la lengua puede afectar a la vida diaria de las personas.

Sin embargo, el hecho de que la sociedad abra continuamente el debate sobre la enseñanza de la lengua no hace más que poner de manifiesto que existe una preocupación real tanto de la administración como de la ciudadanía por esta cuestión.

La iniciativa de que la Unión Europea apueste decididamente por un enfoque comunicativo de la lengua (al menos desde la Resolución del Consejo de 31 de marzo de 1995, publicada en el boletín oficial de la Unión Europea C207 de 12 de agosto) ha resultado, efectivamente, decisiva a la hora de clarificar las metas y los objetivos concretos del aprendizaje.

Sin duda, el auge de los estudios, conferencias e iniciativas de implantación de metodologías integradas de lengua y contenidos en el sistema educativo favorece también que el público en general decida entrar en el debate sobre la educación bilingüe. Pero lo habitual en estas iniciativas es que se centren en el "cómo" de la cuestión: cómo se evalúa, cómo se mide la competencia, cómo se enfoca el aprendizaje, cómo debe actuar el profesor, cuál debe ser la actitud del aprendiz, cómo deben actuar los gobernantes, cómo deben reflejarse los objetivos lingüísticos en las legislaciones estatales, y un largo etcétera.

Como decía, es difícil encontrar razones que no vayan más allá de objetivos políticos a corto plazo. Uno de estos porqués de la enseñanza de las lenguas viene dado por los informes y comunicaciones redactados por la División de Política Lingüística de la Comisión Europea y que se pueden encontrar fácilmente en las páginas web oficiales de dicho organismo. De todas formas, cualquier ciudadano de la Unión parece estar convencido de la necesidad del aprendizaje de la lengua extranjera, tal y como se afirma en el resumen para España del Eurobarómetro sobre las lenguas (Eurydice, 2006:1).

De este modo, el hecho de que el papel del idioma extranjero se convierta en incuestionable pone de manifiesto el grado de convencimiento que demuestran los europeos sobre la necesidad de conocer lenguas y el grado de eficacia que se espera de una política lingüística ejecutada por las autoridades comunitarias. Por su lado, también resulta significativo que las razones últimas de los europeos, aparte de la tolerancia intercultural, se distribuyan entre el trabajo, los estudios y el ocio. De cualquiera de las maneras, según el informe estadístico "Foreign language teaching in schools in Europe" (2001:1), citando al Eurobarómetro de ese año, se indica que en aquel momento ya el $71 \%$ de los europeos y el $93 \%$ de los padres creían que todo el mundo debería hablar por lo menos una lengua comunitaria aparte de la propia y que aprenderla era algo importante.

Aunque no tengo nada que objetar al hecho de que el aprendizaje de una lengua persiga un objetivo utilitario del tipo que sea en un momento dado, no es menos cierto que una política educativa global no solo debe apuntar a objetivos utilitarios sino hacia un enfoque holístico, o sea, la formación integral de la persona.

En este artículo quisiera poner de manifiesto la necesidad de la búsqueda de una razón pedagógica para el aprendizaje de lenguas extranjeras, al mismo tiempo que apuesto también por una alternativa de origen crítico cuyo objetivo último sea un cambio de mentalidad en el profesorado, encaminado a convertir la lengua en un vehículo para la cohesión y justicia social. 


\section{El bILINGÜISMo FATALISTA Y EL VALOR FETICHE DE LA LENGUA}

Según Paulo Freire, el fatalismo, en términos pedagógicos, es una actitud nacida de una ideología en cuya raíz se encuentra la falta de esperanza de cambio social y que, por tanto, acepta como inevitable la situación presente, de forma tal que se explica la existencia humana en términos de determinación de la historia, como pieza de un engranaje que a su vez forma parte de una cosmovisión mecanicista que dice conocer o predecir el futuro (Freire, 2010:61-67). El fatalismo educativo es pensar que la educación sigue principios de linealidad (Robinson, 2011:58-59) en virtud de los cuales la educación se planifica con intención de asentar las bases necesarias para un futuro que se prevé competitivo y exigente en cuanto a la demanda de una serie de perfiles humanos que han de cubrir ciertos puestos de trabajo.

En el campo del aprendizaje de las lenguas extranjeras, esta actitud respondería a la demanda de una lengua en función de su utilidad a la hora de encontrar un empleo, es decir, motivada por la futura proyección de los alumnos en un mercado laboral. En la actualidad, existe una idea generalizada de que las lenguas favorecen la empleabilidad del futuro titulado, de forma que se subordina la educación a las leyes de un mercado hipotético. Las legislaciones que favorecen e impulsan el desarrollo de programas de bilingüismo en centros públicos y privados contienen, en sus prefacios y motivaciones, afirmaciones que cabrían dentro de esta definición de fatalismo histórico. Por ejemplo, como ilustración de lo que venimos comentando, en el prefacio de la Orden 5958/2010, de 7 de diciembre, publicada en el Boletín Oficial de la Comunidad de Madrid y por la que se regulan los colegios públicos bilingües se dice (el subrayado es mío):

El dominio de este idioma [inglés] es una herramienta imprescindible para que nuestros alumnos alcancen, gracias a un programa basado en la igualdad de oportunidades en una escuela pública de calidad, una formación que les permita competir en el ámbito laboral en las mejores condiciones y lograr un excelente futuro personal y profesional.

En este texto se pone de manifiesto que en el espíritu del legislador hay una subordinación total al mercado laboral futuro, a pesar de la incertidumbre que conlleva semejante actitud. Este fenómeno es lo que Freire (2009:25) también denominaba "intransitividad de conciencia", es decir, una actitud mediante la cual la persona se circunscribe a un ámbito muy reducido de su existencia (en este caso su empleabilidad hipotética), de manera que a la persona no se le deja ver toda la globalidad y riqueza que reporta el conocimiento o, yendo un paso más allá, la riqueza que supone pensar en todas aquellas cosas desconocidas a las que nos enfrenta un nuevo conocimiento. Se corre el riesgo, así, de anular una disposición a conocer y convertirla en una actitud pasiva ante la realidad, que se conoce, además, de forma parcial e inauténtica, en tanto que se falsea el objetivo real del proceso educador.

Un segundo ejemplo de esta misma cuestión, pero a un nivel legislativo superior, se encuentra en el preámbulo XIII de la exposición de motivos de la Ley Orgánica 8/2013 para la Mejora de la Calidad de la Educación (LOMCE) de 9 de diciembre, donde se dice (el subrayado es mío):

La ley apoya decididamente el plurilingüismo, redoblando los esfuerzos para conseguir que los estudiantes se desenvuelvan con fluidez en al menos una pri- 
mera lengua extranjera, cuyo nivel de comprensión oral y lectora y de expresión oral y escrita resulta decisivo para favorecer la empleabilidad y las ambiciones profesionales, y apuesta decididamente por la incorporación curricular de una segunda lengua extranjera.

Por lo que se entiende que la (única, ya que no se nombran más) motivación para la introducción de una lengua extranjera en el currículo viene determinada por la asunción de que el mercado demandará trabajadores con una cualificación concreta que el legislador es capaz de adivinar y a cuya inevitabilidad se somete.

En ese trasfondo ideológico se identifica la competitividad con el éxito personal y se aduce que las leyes educativas están para responder a las ambiciones profesionales, sesgando así la naturaleza holística de la persona y reduciéndola a un factor de producción narcisista. En términos críticos se hablaría de una conciencia propia de una sociedad cerrada, vuelta sobre sí misma, en la que los problemas no se contemplan si no están dentro de los límites de la propia experiencia vital, de tal forma que los desafíos que plantea la realidad externa son observables pero no se capta la verdadera red causal que los provoca y, por lo tanto, se reacciona contra ellos desde la conciencia mágica fatalista, que acepta, como decíamos anteriormente, el futuro o los resultados del mercado de trabajo como hechos consumados.

Estoy de acuerdo con el análisis de Bourdieu (2012:49) cuando afirma que la relación dialéctica entre escuela y mercado en el siglo diecinueve permitió la unificación lingüística mediante la introducción de unos estándares nacionales en cada Estado, desplazando así las lenguas locales y convirtiéndolas en lenguas marginales, con distinto valor social. Es este valor de mercado el que hace — siguiendo con la metáfora económica de Bourdieu - que unas lenguas alcancen un valor subjetivo mayor que otras. Esto sería lo que Marx denominaría valor fetiche de un objeto, o sea, el otorgamiento de un valor de mercado a algo que en realidad podría no tenerlo.

En otras palabras, las lenguas se defienden por su valor económico o de poder, no por su capacidad, por ejemplo, comunicativa, cultural o intrínseca de cualquier otro tipo.

El hecho de que el Estado español o la Unión Europea - no solo- hayan otorgado a la lengua inglesa el poder de generar expectativas de empleo produce que el valor subjetivo de esa lengua entre los ciudadanos crezca, al mismo tiempo que las lenguas nacionales, lógicamente, disminuyen en su "cotización" de poder simbólico. Al entrar las lenguas a cotizar en un mercado real, aunque sea subjetivo, siempre habrá quien posea más de ese bien cotizado (elevado a la categoría de capital cultural) y habrá quien posea menos, ya que esa es la lógica de cualquier mercado. Entonces se debe suponer que quien obtenga más de ese bien disfrutará en el futuro de una mayor empleabilidad. La lengua, por tanto, se convierte en un factor de posible desigualdad social.

\section{LA COMUNIDAd LINGÜÍSTICA Y EL PROBLEMA DE LA MULTICULTURALIDAd}

Con la expansión de una lengua se producen dos fenómenos complementarios. El primero es el de la imposición. Las lenguas extranjeras, especialmente el inglés en nuestros días, vive un proceso muy similar al de la implantación de las lenguas oficiales. Estas son lenguas cuyo soporte principal es el Estado; en el caso de la lengua inglesa, además, son 
las instituciones internacionales, empresas multinacionales y entidades supranacionales. La lengua oficial es la variedad de la lengua comúnmente aceptada mediante un proceso de estandarización y de normativización, a la que todo hablante debe referirse y cuya variedad supone la "norma" que sirve de contraste para los usos no aceptables. Este es un proceso al que Bourdieu (2012:50-51) llama "dominación simbólica". Esta dominación no se produce, sin embargo, por la imposición forzada o violenta de una lengua sino con la aquiescencia por parte del dominado. Aunque hay una fuerza externa que lo impone, hay también una sumisión voluntaria.

El otro proceso que se da simultáneo al anterior es el de la ampliación de la comunidad de hablantes. Es decir, cuando aprendemos una lengua también empezamos a formar parte de la comunidad de sus hablantes; de ese modo, la lengua queda aprehendida y, por tanto, es susceptible de sufrir transformaciones, procesos de pidginización, por parte de todos aquellos que la hablan.

El fenómeno del inglés actual, por el contrario de lo que suele suceder, no está relacionado con una comunidad lingüística que se impone a otra, sino que lo hace en una variedad estándar cuyos hablantes no forzadamente son nativos de la lengua pero que la utilizan en función de su rol en el mercado. El inglés es contemplado no como la lengua de una comunidad sino como el código necesario para acceder a una serie de privilegios económicos.

Sin embargo, a pesar de esta situación, la mayoría de europeos entiende, seguramente de forma inconsciente, que las lenguas son primordialmente expresiones de una comunidad cultural. Así lo demuestra el hecho de que el ocio o el turismo representan buena parte de la motivación para aprender una lengua (Eurydice, 2008), aparte de las razones meramente laborales. El peso, por tanto, de lo cultural sobre lo normativo y lo económico es un factor a tener en cuenta. No obstante, un fenómeno de imposición como este, a diferencia de lo que ha pasado en otras etapas históricas donde la lengua se imponía desde el poder por hablantes que participaban en ese poder, se basa en una variedad que no va ligada directamente a una sola cultura concreta. En otras palabras no se impone el inglés de los británicos o de los americanos, sino una variedad internacional estandarizada cuyo fin primordial es la de entenderse en un mercado globalizado.

En España, país con algo más de un cuarto de su población bilingüe (Ethnologue, 2013), aunque no forzadamente en una lengua extranjera, se ha planteado la introducción de la lengua inglesa poniendo en práctica las recomendaciones de la Unión Europea mediante planes de bilingüismo impulsados por las diferentes comunidades autónomas, con lo cual, puedo afirmar sin temor a equivocarme que la amplia mayoría de los estudiantes españoles tienen acceso a la lengua en el aula.

Sin embargo, no hace falta poner en evidencia que el aula es para la gran mayoría de estos alumnos el único contexto donde la lengua inglesa es significativa, de forma tal que con respecto a aquellos alumnos que sí puedan tener contacto con nativos de la lengua de un modo u otro, lo cierto es que se produce en la realidad una diferenciación social en virtud del dominio de la lengua, un fenómeno, desde el punto de vista pedagógico, muy similar al expuesto por V. Unamuno (2003:90) en relación a la enseñanza del catalán a grupos de alumnos marginales, o sea, que no se progresa en la lengua objeto de estudio porque el contexto del alumno no tiene como lengua vehicular la misma que la del colegio, aunque en España el 83,9\% de la población escolar recibe su instrucción en la misma lengua que usan en casa (Eurydice, 2008:20). Algunos programas de bilingüismo, públicos o de iniciativa 
privada, siguen más un modelo de submersión, es decir la sustitución de las lenguas "débiles" por la dominante (Grosjean, 2012:229-236), en vez de desarrollar programas de inmersión, o sea, utilizar la lengua extranjera como vehicular junto con la(s) otra(s) de los alumnos, con el objeto de alcanzar la meta de ser realmente bilingüe (si entendemos bilingüismo en su concepción menos laxa). Este uso está de acuerdo con el principio de complementariedad enunciado por Grosjean (2012:29) en virtud del cual las personas bilingües adquieren sus lenguas con diferentes propósitos, en diferentes dominios de la vida para comunicarse con gente diferente, por lo que a diferentes aspectos de la vida corresponden diferentes lenguas.

Esta realidad tiene una consecuencia pedagógica directa. La pedagogía actual no solo vela por el traspaso de conocimientos en forma de datos, sino también de valores, y para ello propone diferentes modelos o enfoques que permiten hacer frente a la realidad de la multiculturalidad (Osuna, 2012:45). Uno de los enfoques que permiten hacer frente a los valores culturales dispares es el de la educación bicultural, tal vez el más ampliamente defendido por la comunidad docente, de forma que los hablantes de una lengua no vean en peligro su propia identidad cultural, al mismo tiempo que asumen valores que acompañan la cultura de la otra lengua, con el fin de entender mejor la vertiente idiomática de la lengua objeto de estudio.

Cuando una cultura está bien definida, como sería por ejemplo la cultura británica, este enfoque sería correcto y deseable. Sin embargo, como decía con anterioridad, actualmente los agentes que expanden la lengua no necesariamente son los miembros nativos de la comunidad lingüística británica, ni tan siquiera anglosajona. Aparte de los datos mencionados de la UE, la realidad española se pone de manifiesto con la motivación legal que he ejemplificado en la introducción, pero no solo. En la I Encuesta Adecco Profesional sobre excelencia de idiomas (2013) muestra que el 53,7\% de los encuestados afirma que se le exigía el conocimiento de una lengua extranjera durante el proceso de selección, de los cuales en el 61,3\% de los casos era el inglés. Por tanto, no se puede afirmar que la lengua venga impuesta por una comunidad de hablantes nativa definida que directamente ostente los mecanismos de poder o control social, sino indirectamente a través de una comunidad dispar multicultural, una amalgama de miembros de diferentes culturas, que utiliza la lengua para fines y usos que no son todos los propios de una comunidad natural de hablantes sino que atienden solo a una parte, mayoritaria o exclusivamente la del intercambio comercial.

Consecuentemente, un enfoque meramente bicultural o de asimilación cultural se quedarían cortos en sus objetivos, pues no hay una cultura definida para usarla de referencia. Sin embargo, por las razones que he aducido en el apartado anterior, un enfoque sociocrítico que busque eliminar en lo posible la asimetría cultural, social y política mediante el desarrollo del nivel de consciencia, sí sería válido, ya que no solo se fija en la cultura en sí misma sino también más ampliamente en los aspectos sociopolíticos, que son precisamente los que están condicionando el aprendizaje de la lengua.

\section{Profundizar en las bases del bilingüismo Crítico}

Frente a la comprensión fatalista de la adquisición de la lengua extranjera y frente al reto de su imposición multicultural se contrapone una manera de entender el fenómeno del bilingüismo que, atendiendo a su esencia pedagógica, se puede denominar crítica y que, según 
su naturaleza funcional y comunicativa, también podemos calificar de dialógica, coincidiendo así con la pedagogía crítica freiriana (Freire, 2009:80). A continuación plantearemos las bases para una elaboración diferente del paradigma de la adquisición de la lengua extranjera.

Para sentar las bases de este paradigma utilizaré como guía y referencia las preguntas, un tanto radicales, que proponía Freire (2010:90) para distinguir un proceso educativo alienante del que no lo es, pero que a pesar de su formulación tan simplificada ayudan a centrar el debate sobre la cuestión que planteo. Las preguntas son: “¿con qué fin estudio? ¿en favor de quién? ¿en contra de qué estudio? ¿en contra de quién estudio? ${ }^{1}$ "

El fin del estudio, desde mi perspectiva, tiene dos vertientes. Por un lado un aspecto relacional, de influencia sobre la realidad circundante y, por otro lado, de búsqueda de los instrumentos intelectuales que permiten al ser humano estar con el mundo y ser consciente de su propia naturaleza y, consecuentemente, de capacitación para conceptualizar la experiencia que el ser humano, en su corporeidad, vive de manera constante. El ser humano, por ser autoconsciente (Fromm, 2011:40) es capaz también de comprender las realidades como cosas distintas a sí mismo, experimentarlas, conceptualizarlas, categorizarlas si fuera necesario y, por último, lexicalizarlas (Johnson, 2008:11-15). El estudio, el acto de estudiar, tiene, por tanto, una razón de ser en el mero hecho del conocimiento de la realidad, pero también en el hecho diferenciador de reafirmar la naturaleza propia del ser humano. En otras palabras, el ser humano, mediante el estudio, profundiza en su autoconciencia. Freire (2010) llega implícitamente a esta misma conclusión cuando apoya los programas de alfabetización de las personas más desfavorecidas, hecho que tiene una especial relevancia para el bilingüismo crítico, como se verá más adelante.

El enfoque crítico que aquí se apoya no es un posicionamiento ni de tipo partidista ni de alineación con corrientes lingüísticas al uso. Más bien, mi intención es proponer un paradigma de reflexión sobre el fenómeno del bilingüismo que impida que la sociedad acate unas ideas o formas de conducta que le vienen dadas sin habérselas cuestionado previamente. Al interrogante sobre el fin del estudio bien podría aplicársele lo afirmado por Horkheimer en relación a la teoría crítica frente a la tradicional (Horkheimer, 2008:223): "Teoría es la acumulación del saber en forma tal que este se vuelva utilizable para caracterizar los hechos de la manera más acabada posible”.

Esto tiene como consecuencia que el conocimiento de la realidad más profunda, a veces mediante la simple reflexión sobre las proposiciones que se aceptan comúnmente (por ejemplo, "el inglés cumplirá tus ambiciones profesionales"), lleve a la relativización de dichas proposiciones. Un bilingüismo crítico vendrá motivado por el intento de superar la tensión que hay entre el individuo que vive en una comunidad y que explora la comunicación con otros individuos de otras comunidades, con la praxis social fundamentada únicamente en relaciones de trabajo. Por tanto, la segunda de las condiciones que postula Freire anunciadas más arriba, "en favor de quién", queda también contestada, ya que la persona en comunidad es el fin del bilingüismo crítico, o sea, en última instancia, como afirmaba el propio Horkheimer (2008:270) el fin remoto es "la supresión de la injusticia social".

La tercera condición freiriana, "en contra de qué estudio" parte del fin que acabamos de enunciar, la superación de las injusticias sociales y la alienación cultural. La adquisición

\footnotetext{
${ }^{1}$ Estas preguntas así planteadas suenan muy radicales y combativas, pero en la pedagogía de Freire, no hay que olvidarlo, el enemigo es la alienación social. En cierto modo estas preguntas contienen un espíritu de lucha intelectual contra la pobreza social, la ignorancia y la explotación, y en ese contexto es como hay que entenderlas.
} 
de la segunda lengua, en el duelo entre el tener y el ser tan propio de la sociedad comercializante actual, se caracteriza por tasar a la persona según el valor de su empleabilidad, falseando así las relaciones interpersonales y uno de sus instrumentos, o sea, el lenguaje. El mensaje socialmente aceptado presupone que la adquisición de unas lenguas añade un valor equis superior al valor que añaden otras lenguas, de forma que hay una jerarquía comercial entre las lenguas. Por lo que queda claro que el objetivo "contra" el que se trabaja es el de una instrucción pobre, de mera asimilación de contenidos, un conocimiento adquirido por otros (Fromm, 2012:138) que no lleva a pensar más o a desarrollar una imaginación activa que sea de verdad un factor productivo para la comunidad.

Por último, tal vez la condición más delicada de expresar en sus justos términos, sea el punto que Freire denomina "en contra de quién". Anteriormente hemos puesto dos ejemplos de legislación que recogen los prejuicios que venimos denunciando, así como referencias a la Unión Europea, pero es difícil captar la intangibilidad de un prejuicio social sino es por medio de estudios estadísticos generales. En el informe de Eurydice "Key Data on Teaching Languages at School in Europe" (2012:103) se ofrecen datos sobre la motivación de los estudiantes en el estudio de una primera y una segunda lengua extranjera. Extrapolamos los porcentajes relevantes en la siguiente tabla:

Tabla I. Motivación de los alumnos en L1 y L2

\begin{tabular}{|c|c|c|}
\hline & L1 & L2 \\
\hline Vida personal & 51 & 21,6 \\
\hline Educación futura & 90,8 & 73,0 \\
\hline Trabajo futuro & 89,8 & 68,7 \\
\hline Obtención de un mejor trabajo & 96,8 & 82,8 \\
\hline
\end{tabular}

La tabla I pone de manifiesto que entre los jóvenes ha calado el mensaje de que la primordial motivación para el aprendizaje de idiomas es la ambición de obtener un mejor trabajo. En términos críticos se diría que vence el "tener" sobre el "ser" (que representaría la vida personal). La variable de "educación futura" es ambigua, ya que puede ser interpretada como parte del "ser", es decir, la voluntad de mejorar en la formación personal, pero también responde al "tener", ya que buena parte de los estudiantes podrían enfocar sus estudios hacia la obtención de un buen trabajo en vez de enfocarlas hacia la realización de su ser personal. Como consecuencia, el bilingüismo crítico actuaría contra aquellos que promueven que la lengua extranjera sea una respuesta refleja a una situación, tal y como lo haría una sociedad alienada.

\subsection{Los fundamentos del paradigma del bilingüismo crítico-dialógico}

El paradigma bilingüe crítico tiene como objeto fundamental la búsqueda de la auténtica causalidad de las realidades humanas. En terminología freiriana habría que hablar del 
paso de la intransitividad a la transitividad, es decir, pasar del estado por el que se actúa en función de condicionamientos irreales, hipotéticos, a un estado en el que los intereses de la persona se amplían a los intereses del otro y superan su mínimo horizonte personal para abrirse a la realidad ajena. Se ve, entonces, que el bilingüismo crítico debe afectar al plano del yo personal por un lado y, por otro, al yo en su entorno social. Aunque en este artículo no puedo desarrollar toda una didáctica, sí voy a avanzar lo que serían los puntos de partida, la epistemología si se quiere, de una posible didáctica crítica de la lengua extranjera basada en los presupuestos mencionados.

Soy consciente de que una didáctica crítica dialógica también fue propuesta por Cummins (2000) para entornos diferentes de integración de minorías lingüísticas con parecidos planteamientos, lo que no impide que esta denominación se pueda usar en entornos donde la lengua extranjera no sea la minoritaria, con sus debidas matizaciones.

En primer lugar, reconocemos la necesidad de subrayar la naturaleza singular, única e individual de la persona ya que vamos a hacer compatible el enfoque crítico con la personalización en educación según la entendía Pierre Faure (Pereira, 1976). Personalización individual, pero no individualista; no se trata de una persona aislada sino interdependiente que no empaña el hecho de ser uno y singular. Bajo este epígrafe de singularidad se entiende la enseñanza como:

- Educar en el "yo me maravillo", el asombro y la curiosidad intelectual, ante los modos de expresión en otra lengua.

- Educar en la plasticidad, o sea, en la disposición a lo nuevo.

- Educar en la problematización de la historia personal, considerando así que la realidad a la que uno se enfrenta se puede resolver conociendo el contexto en cada momento, descifrando las claves que inciden en el ciclo vital.

- Educar en la consciencia de la finitud de la persona, de forma que la comunicación con los demás sea vista como el proceso natural para el crecimiento propio.

- Educar en el carácter experimental de la lengua; una apertura a experimentar y conceptualizar las experiencias humanas a través del nuevo léxico.

- Educar en la dimensión creativa del lenguaje.

En segundo lugar, el aprendiz de lenguas debe darse cuenta de que es un ser libre y autónomo como creador de cultura. Una persona sería incapaz de dar ese paso si se le impide desde fuera su capacidad de expresión, o esta es mínima. Precisamente, la alfabetización (el aprendizaje y dominio de la primera lengua) es clave para concienciar al individuo de su ubicación en la sociedad. En la sociedad contemporánea, ganar en autonomía con respecto de las lenguas extranjeras exige un proceso de post-alfabetización, una nueva alfabetización, o sea un nuevo proceso de concienciación de la persona del papel que tiene en una sociedad globalizada que utiliza una lengua estándar como vehículo de expresión. Ese proceso de segunda alfabetización le permitirá o facilitará tener voz como ciudadano en estratos de una sociedad que de otras formas le aparecería como cerrada y en cierto modo mágica. Con este fin el docente debe plantearse:

- Educar en la toma de decisiones sobre lo que se necesita y se quiere aprender de la lengua extranjera.

- Educar en la pluralidad, es decir, entender la realidad como desafío, en la que el proceso educativo es la búsqueda de la mejor respuesta. 
- Educar en la profunda interpretación de los problemas que ofrece la realidad lingüística para poder encontrar una salida satisfactoria.

- Educar en la contemplación y la observación de la realidad para comprenderla mejor sin emitir juicios infundados.

- Educar en el abandono de prejuicios en el análisis de cuestiones que atañen a la comunicación humana, metodologías de enseñanza o enfoques.

- Educar para la asunción de responsabilidades, especialmente a la hora de poner en práctica las distintas funciones del lenguaje, de manera que el aprendiz de la lengua sea consciente que comunicar es también asumir los principios de veracidad y justicia.

En tercer lugar, la sociedad tiene un papel muy relevante en el bilingüismo crítico. Primordialmente la educación identifica los contextos de la realidad y ayuda a la persona a averiguar su sentido personal en el mundo. Tiene, por consiguiente un carácter instrumental. En el caso del aprendizaje de la lengua, este carácter lo define la dimensión comunicativa, que es, por definición, social. Este principio de apertura al otro es a la vez la base y el objetivo no solo de todo el sistema educativo sino especialmente del bilingüismo crítico. El docente trabajaría la dimensión social del aprendiz de lenguas al:

- Educar desde la perspectiva del otro. El docente puede hacer ver los puntos de vista distintos dentro de la realidad del discurso y su materialización en las estructuras y funciones del lenguaje.

- Educar en el diálogo y evitar la polémica.

- Educar para la integración de la palabra y la experiencia, no para entender la adquisición de la lengua extranjera como un mero depósito de datos gramaticales inconexos.

- Educar en la expresión espontánea.

- Educar en la participación.

- Educar en la conciencia performativa, o sea, que la expresión produce cambios en la realidad.

- Educar con la intención de buscar el desarrollo del otro, sin buscar el propio interés.

- Educar en la dimensión política del lenguaje, en su valor igualador y democratizador, ya que el acceso al vehículo de comunicación del poder, el aprendiz de la lengua extranjera deja de ser reflejo de un modelo importado sino en actor y artífice de una nueva evolución del lenguaje.

Cada una de estas tres dimensiones o principios requeriría un desarrollo didáctico posterior y un alto grado de matización que se alejan del propósito inmediato de este artículo, sin embargo, creo haber señalado hasta aquí lo que podría representar una primera fase del camino que una doble concepción crítica y dialógica puede aportar al currículo de lengua extranjera. No obstante, soy consciente de que no se debe perder la perspectiva del aula en ningún momento y que una sistematización ulterior no solo es deseable sino que es necesaria. 


\section{Conclusiones}

El ciudadano europeo no anglosajón alfabetizado desde la infancia se enfrenta a la realidad lingüística foránea forzado no por su contexto inmediato, que sigue siendo primordialmente monolingüe o bilingüe de otra lengua cooficial, sino por un contexto remoto que los poderes económicos y políticos sitúan en un hipotético futuro. El ciudadano, entonces, acepta de manera ingenua la imposición mercantilista dotando a la lengua extranjera de un poder mágico, donde su conocimiento abre las puertas a la supervivencia individual futura. La dimensión social del lenguaje queda entonces subordinada al uso individualista, intransitivo, y al interés particular. Esta visión de la lengua empobrece e impide el desarrollo de la persona.

Pensar en términos de bilingüismo crítico supone invertir esta situación. Implica hacer crecer la dimensión personal sin olvidar la social. Implica renunciar a ver en el otro a un competidor por un puesto de trabajo o lugar en la sociedad y pasar a considerarlo como agente del crecimiento propio y verse a uno como agente de crecimiento del otro. Implica dar prioridad a la creatividad, la expresión y la construcción del conocimiento. Implica, en definitiva, entender que la lengua no es un capital acumulable en grandes cantidades cuya inversión produce beneficios en el largo plazo, sino un instrumento para la comunicación y transmisión de valores que produce un mayor conocimiento del mundo, de la cultura y del hombre. Es, en definitiva, un modo de devolverle a la lengua su carácter genuino sin contaminaciones mercantilistas.

\section{REFERENCIAS BIBLIOGRÁFICAS}

ADECCO. (2013). "I Encuesta Adecco Profesional sobre exigencia de idiomas", disponible en http://www.adecco.es/_data/NotasPrensa/pdf/506.pdf, consultado el 5 de diciembre de 2013. Bordieu, P. (2012). Language and Symbolic Power. Cambridge: Polity Press.

Cummins, J. (2000). Language, Power and Pedagogy. Bilingual Children in the Crossfire. Clevedon: Multilingual Matters Ltd.

Eurydice. (2006). Los europeos y las lenguas. Bruselas: Eurydice European Unit, disponible en http://ec.europa.eu/spain/pdf/eurobar_lenguas_es.pdf, consultado el 1 de noviembre de 2013.

Eurydice. (2008). Key Data on Teaching Languages at School in Europe 2008. Bruselas: Eurydice European Unit, disponible en http://eacea.ec.europa.eu/education/eurydice/documents/ key_data_series/095EN.pdf, consultado el 1 de noviembre de 2013.

Eurydice. (2012). Key Data on Teaching Languages in Europe 2012. Bruselas: Eurydice European Unit, disponible en http://eacea.ec.europa.eu/education/eurydice/documents/key_data series/143EN.pdf, consultado el 1 de noviembre de 2013.

Freire, P. (2008). El grito manso. Buenos Aires: Siglo Veintiuno Editores.

Freire, P. (2009). La educación como práctica de la libertad. Buenos Aires: Siglo Veintiuno Editores.

Freire, P. (2010). Pedagogía de la indignación. Madrid: Morata

Fromm, E. (2011). El amor a la vida. Madrid: Paidós.

Fromm, E. (2012). Del tener al ser. Madrid: Paidós.

Grosjean, F. (2010). Bilingual. Life and Reality. Cambridge: Harvard University Press. 
Horkheimer, M. (2008). Teoría crítica. Buenos Aires: Amorrortu.

Johnson, J. (2008). The Meaning of the Body. Aesthetics of Human Understanding. Chicago: Chicago University Press.

Lorenzo, F., Trujillo, F. y Vez, J.M. (2011). Educación bilingüe. Integración de contenidos y segundas lenguas. Madrid: Síntesis.

Osuna Nevado, C. (2012). "En torno a la educación intercultural. Una revisión crítica", in Revista de Educación, 358: 38-58.

Pereira, N. (1976). Educación personalizada: un proyecto pedagógico en Pierre Faure. Madrid: Narcea.

Robinson, K. (2011). Out of our Minds. Learning to be Creative. Chichester: Capstone Publishing, Ltd.

Unamuno, V. (2003). Lengua, escuela y diversidad sociocultural. Hacia una educación lingüística crítica. Barcelona: Graó. http://www.ethnologue.com, consultado el 3 de noviembre de 2013. 